\title{
General Common Fixed Point Theorems for Occasionally Weakly Compatible Mapppings
}

\author{
P. SrikanthRao and D. Bharathi \\ Padmasri Dr. B. V. Raju Institute of Technology,Narsapur, Medak District, A.P, India.
}

\begin{abstract}
The Purpose of this paper is to prove common fixed point theorems in metric space by using occasionally weakly compatible six self mappings satisfying the implicit relation which unify and generalize most of the existing relevant fixed point theorems.
\end{abstract}

2000 Mathematics Subject Classification : 54H25, 47H1O

Keywords: Compatible, Weakly compatible, Occasionally Weakly Compatible and implicit relations.

\section{Introduction}

The concept of the commutativity has been generalized in several ways. For this, Sessa, S. [17] has introduced the concept of weakly commuting. Obviously two commuting mappings are weekly commuting but not conversely as given in [17]. Gerald Jungck [4] initiated the concept of compatibility.A weakly commuting pair is compatible but not conversely as given in Jungck [4]. In the later years the concept of compatibility is further generalized in many ways. G. Jungck and P.P. Murthy and Y.J. Cho [5] introduced the concept of compatible mappings of type (A) and they gave some examples to show that compatible maps of type (A) need not be compatible mappings.Extending type (A) mappings H.K.Pathak and M.S.Khan [13] introduced the concept of compatible mappings of type (B) and they gave some examples to show that compatible maps of types (B) need not be compatible mappings of type (A). In 1996,H.K.Pathak, Y.J.Cho, S.S. Chang and S.M.Kang[11] introduced the concept of compatible mappings of type(P) and they gave some examples to show that compatible mappings of type $(\mathrm{P})$ need not be compatible mappings, compatible mappings of type $(\mathrm{A})$, compatible mappings of type(B).In 1998, H.K.Pathak, Y.J.Cho, S.M.Kang and B.Madharia [12] introduced another extension of compatible mappings of type (A) in normed spaces called compatible mappings of type (C) and with some examples they compared these mappings with compatible maps.From the propositions given in [4], [5], [11], [12],[13] we observe that the concept of compatible, compatible mappings of type (A), compatible mappings of type (B),compatible mappings of type $(\mathrm{P})$ and compatible mappings of type (C) are equivalent when $\mathrm{S}$ and $\mathrm{T}$ are continuous. They are independent if the functions are discontinuous. It has been known from the paper of Kannan [8] that there exists maps that have a discontinuity in the domain but which have fixed points. Moreover, the maps involved in every case were continuous at the fixed point. In 1998, Jungck and Rhoades [6] introduced the notion of weakly compatible and showed that compatible maps are weakly compatible but not conversely. Recently in 2006 Jungck and Rhoades [7] introduced occasionally weakly compatible maps(owc) which is more general among the commutativity concepts. The main purpose of this paper is to extend the results of [7] and [15].

\section{Preliminaries}

Definition 2.1. Let $S$ and $T$ be mappings from a metric space (X, d) into itself. The mappings $S$ and $T$ are said to be

$$
\text { Compatible if } \lim _{\mathbf{n} \rightarrow \infty} \mathrm{d}\left(\mathrm{STx}_{\mathrm{n}}, \mathrm{TSx}_{\mathrm{n}}\right)=0
$$

i) Compatible of type(A) if $\lim _{n \rightarrow \infty} d\left(\operatorname{STx}_{n}, \operatorname{TTx}_{n}\right)=0$ and

$$
\lim _{n \rightarrow \infty} d\left(\operatorname{TSx}_{n}, S x_{n}\right)=0
$$

(ii) Compatible of type(B) if

$$
\lim _{\mathbf{n} \rightarrow \infty} d\left(\operatorname{STx}_{n}, \operatorname{TTx}_{n}\right) \leq \frac{1}{2}\left[\lim _{\mathbf{n} \rightarrow \infty} d\left(\operatorname{STx}_{n}, \operatorname{St}\right)+\lim _{\mathbf{n} \rightarrow \infty} d\left(\operatorname{St}, \operatorname{SSx}_{\mathbf{n}}\right)\right] \text { and }
$$

$$
\lim _{n \rightarrow \infty} d\left(\operatorname{TSx}_{n}, \operatorname{SSx}_{n}\right) \leq \frac{1}{2}\left[\lim _{n \rightarrow \infty} d\left(\mathrm{TSx}_{n}, T \mathrm{t}\right)+\lim _{\mathbf{n} \rightarrow \infty} d\left(T t, \operatorname{TTx}_{n}\right)\right]
$$

$$
\text { Compatible of type }(P) \text { if } \lim _{n \rightarrow \infty} d\left(\operatorname{SSx}_{n}, \operatorname{TTx}_{n}\right)=0
$$


(v)

$$
\begin{aligned}
& \text { Compatible of type(C) if } \\
& \lim _{\mathbf{n} \rightarrow \infty} \mathrm{d}\left(\mathrm{STx}_{\mathrm{n}}, \mathrm{TTx}_{\mathrm{n}}\right) \leq \frac{1}{3}\left[\lim _{\mathbf{n} \rightarrow \infty} \mathrm{d}\left(\mathrm{STx}_{\mathrm{n}}, \mathrm{St}\right)+\lim _{\mathbf{n} \rightarrow \infty} \mathrm{d}\left(\mathrm{St}, \mathrm{SSx}_{\mathrm{n}}\right)+\lim _{\mathbf{n} \rightarrow \infty} \mathrm{d}\left(\mathrm{St}, \mathrm{TTx}_{\mathrm{n}}\right)\right] \text { and } \\
& \lim _{\mathbf{n} \rightarrow \infty} \mathrm{d}\left(\mathrm{TSx}_{\mathrm{n}}, \mathrm{SSx}_{\mathrm{n}}\right) \leq \frac{1}{3}\left[\lim _{\mathbf{n} \rightarrow \infty} \mathrm{d}\left(\mathrm{TSx}_{\mathrm{n}}, \mathrm{Tt}\right)+\lim _{\mathbf{n} \rightarrow \infty} \mathrm{d}\left(\mathrm{Tt}, \mathrm{TTx}_{\mathrm{n}}\right)+\right. \\
& \left.\qquad \lim _{\mathbf{n} \rightarrow \infty} \mathrm{d}\left(\mathrm{Tt}, \mathrm{SSx}_{\mathrm{n}}\right)\right]
\end{aligned}
$$

when ever $\left\langle x_{n}\right\rangle$ is a sequence in $X$ such that $\lim _{n \rightarrow \infty} S_{x_{n}}=\lim _{n \rightarrow \infty} T x_{n}=t \quad$ for some $t \in X$.

Definition 2.2. [6]. A pair of maps $\mathrm{T}$ and $\mathrm{S}$ is called weakly compatible pair if they commute at coincidence points.

Definition 2.3. [7]. Two self maps $S$ and $T$ on a set $X$ are said to be occasionally weakly compatible(owc) if and only if there is a point $\mathrm{x} \in \mathrm{X}$ which is a coincidence point of $\mathrm{S}$ and $\mathrm{T}$ at which $\mathrm{S}$ and $\mathrm{T}$ commute. i.e., there exists a point $\mathrm{x} \in \mathrm{X}$ such that $\mathrm{Sx}=\mathrm{Tx}$ and $\mathrm{STx}=\mathrm{TSx}$.

\section{Implicit Relations.}

Let $F$ be the set of all continuous functions $\mathrm{F}: \mathrm{R}_{+}^{6} \rightarrow \mathrm{R}$ satisfying the following conditions:

(3.1) $\mathrm{F}$ is non-increasing in variables $\mathrm{t}_{5}$ and $\mathrm{t}_{6}$.

(3.2) there exists $h \in(0,1)$ such that for $u, v \geq 0$ with

(3.3) $\mathrm{F}(\mathrm{u}, \mathrm{v}, \mathrm{v}, \mathrm{u}, \mathrm{u}+\mathrm{v}, 0) \leq 0$

(3.4) $\mathrm{F}(\mathrm{u}, \mathrm{v}, \mathrm{u}, \mathrm{v}, 0, \mathrm{u}+\mathrm{v}) \leq 0$ implies $\mathrm{u} \leq \mathrm{h}$.v.

(3.5) $\mathrm{F}(\mathrm{u}, \mathrm{u}, 0,0, \mathrm{u}, \mathrm{u})>0$ for all $\mathrm{u}>0$.

The following examples of such functions F satisfying (3.1), (3.2), (3.3), (3.4) and (3.5) are available in [15] with verifications and other details.

Example 3.6: Define $F\left(t_{1}, t_{2}, \ldots, t_{6}\right): R_{+}^{6} \rightarrow R$ as

$$
\mathrm{F}\left(\mathrm{t}_{1}, \mathrm{t}_{2}, \ldots, \mathrm{t}_{6}\right)=\mathrm{t}_{1}-\mathrm{k} \max \left\{\mathrm{t}_{2}, \mathrm{t}_{3}, \mathrm{t}_{4}, \frac{1}{2}\left(\mathrm{t}_{5}+\mathrm{t}_{6}\right)\right\} \text {, where } \mathrm{k} \in(0,1) \text {. }
$$

Example 3.7: Define $F\left(t_{1}, t_{2}, \ldots, t_{6}\right): R_{+}^{6} \rightarrow R$ as

$\mathrm{F}\left(\mathrm{t}_{1}, \mathrm{t}_{2}, \ldots, \mathrm{t}_{6}\right)=\mathrm{t}_{1}^{2}-\mathrm{t}_{1}\left(\alpha \mathrm{t}_{2}+\beta \mathrm{t}_{3}+\gamma \mathrm{t}_{4}\right)-\eta \mathrm{t}_{5} \mathrm{t}_{6}$

where $\alpha>0 ; \beta, \gamma, \eta \geq 0 ; \alpha+\beta+\gamma<1$ and $\alpha+\eta<1$.

Example 3.8: Define $F\left(t_{1}, t_{2}, \ldots, t_{6}\right): R_{+}^{6} \rightarrow$ Ras

$\mathrm{F}\left(\mathrm{t}_{1}, \mathrm{t}_{2}, \ldots, \mathrm{t}_{6}\right)=\mathrm{t}_{1}^{3}-\alpha \mathrm{t}_{1}^{2} \mathrm{t}_{2}-\beta \mathrm{t}_{1} \mathrm{t}_{3} \mathrm{t}_{4}-\gamma \mathrm{t}_{5}^{2} \mathrm{t}_{6}-\eta \mathrm{t}_{5} \mathrm{t}_{6}^{2}$,

where $\alpha>0 ; \beta, \gamma, \eta \geq 0 ; \alpha+\beta<1$ and $\alpha+\gamma+\eta<1$.

Example 3.9: Define $F\left(t_{1}, t_{2}, \ldots, t_{6}\right): R_{+}^{6} \rightarrow R$ as

$$
\mathrm{F}\left(\mathrm{t}_{1}, \mathrm{t}_{2}, \ldots, \mathrm{t}_{6}\right)=\mathrm{t}_{1}^{3}-\alpha \frac{\mathrm{t}_{3}^{2} \mathrm{t}_{4}^{2}+\mathrm{t}_{5}^{2} \mathrm{t}_{6}^{2}}{1+\mathrm{t}_{2}+\mathrm{t}_{3}+\mathrm{t}_{4}} \text {, where } \alpha \in(0,1) \text {. }
$$

Example 3.10: Define $F\left(t_{1}, t_{2}, \ldots, t_{6}\right): R_{+}^{6} \rightarrow R$ as

$$
\mathrm{F}\left(\mathrm{t}_{1}, \mathrm{t}_{2}, \ldots, \mathrm{t}_{6}\right)=\mathrm{t}_{1}^{2}-\alpha \mathrm{t}_{2}^{2}-\beta \frac{\mathrm{t}_{5} \mathrm{t}_{6}}{1+\mathrm{t}_{3}^{2}+\mathrm{t}_{4}^{2}} \text {, where } \alpha>0, \beta \geq 0 \text { and } \alpha+\beta<1 \text {. }
$$

Example 3.11: Define $\mathrm{F}\left(\mathrm{t}_{1}, \mathrm{t}_{2}, \ldots, \mathrm{t}_{6}\right): \mathrm{R}_{+}^{6} \rightarrow \mathrm{R}$ as

$$
F\left(t_{1}, t_{2}, \ldots, t_{6}\right)=\left\{\begin{array}{c}
t_{1}-a_{1} \frac{t_{3}^{2}+t_{4}^{2}}{t_{3}+t_{4}}-a_{2} t_{2}-a_{3}\left(t_{5}+t_{6}\right), \text { if } t_{3}+t_{4} \neq 0 \\
t_{1}, \text { if } t_{3}+t_{4}=0
\end{array}\right.
$$

(3.1): Obvious.

Where $a_{i} \geq 0$ with at least one $a_{i}$ non zero and $a_{1}+a_{2}+2 a_{3}<1$.

(3.2) ((3.3)): Let $u>0, F(u, v, v, u, u+v, 0)=u-a_{1}\left(v^{2}+u^{2}\right) /(v+u)-a_{2} v-a_{3}(u+v) \leq 0$. If $u \geq v$, then $\mathrm{u} \leq\left(\mathrm{a}_{1}+\mathrm{a}_{2}+2 \mathrm{a}_{3}\right) \mathrm{u}<\mathrm{u}$ which is a contradiction. Hence $\mathrm{u}<\mathrm{v}$ and $\mathrm{u} \leq \mathrm{hv}$ where $\mathrm{h} \in(0,1)$.

(3.4): Similar argument as in (3.3).

(3.5): $\mathrm{F}(\mathrm{u}, \mathrm{u}, 0,0, \mathrm{u}, \mathrm{u})=\mathrm{u}>0$ for all $\mathrm{u}>0$.

We also add the following examples [16] without verification.

Example 3.12: Define $F\left(t_{1}, t_{2}, \ldots, t_{6}\right): R_{+}^{6} \rightarrow$ Ras

$$
\mathrm{F}\left(\mathrm{t}_{1}, \mathrm{t}_{2}, \ldots, \mathrm{t}_{6}\right)=\left\{\begin{array}{c}
\mathrm{t}_{1}-\alpha \mathrm{t}_{2}-\frac{\beta \mathrm{t}_{3} \mathrm{t}_{4}+\gamma \mathrm{t}_{5} \mathrm{t}_{6}}{\mathrm{t}_{3}+\mathrm{t}_{4}}, \text { if } \mathrm{t}_{3}+\mathrm{t}_{4} \neq 0 \\
\mathrm{t}_{1}, \text { if } \mathrm{t}_{3}+\mathrm{t}_{4}=0
\end{array}\right.
$$

Where $\alpha, \beta, \gamma \geq 0$ such that $1<2 \alpha+\beta<2$.

Example 3.13: Define $F\left(t_{1}, t_{2}, \ldots, t_{6}\right): R_{+}^{6} \rightarrow R$ as 
$F\left(t_{1}, t_{2}, \ldots, t_{6}\right)=t_{1}-a_{1} t_{2}-a_{2} t_{3}-a_{3} t_{4}-a_{4} t_{5}-a_{5} t_{6}$ where $\sum_{i=1}^{5} a_{i}<1$

Example 3.14: Define $F\left(t_{1}, t_{2}, \ldots, t_{6}\right): R_{+}^{6} \rightarrow$ Ras

$$
\begin{aligned}
& F\left(t_{1}, t_{2}, \ldots, t_{6}\right)=t_{1}-\alpha\left[\beta \max \left\{t_{2}, t_{3}, t_{4}, \frac{1}{2}\left(t_{5}+t_{6}\right)\right\}+\right. \\
& \left.(1-\beta)\left[\max \left\{t_{2}^{2}, t_{3} t_{4}, t_{5} t_{6}, \frac{t_{3} t_{6}}{2}, \frac{t_{4} t_{5}}{2}\right\}\right]^{\frac{1}{2}}\right], \text { where } \alpha \in(0,1) \text { and } 0 \leq \beta \leq 1 .
\end{aligned}
$$

Example 3.15: Define $F\left(t_{1}, t_{2}, \ldots, t_{6}\right): R_{+}^{6} \rightarrow R$ as

$$
\begin{aligned}
& \mathrm{F}\left(\mathrm{t}_{1}, \mathrm{t}_{2}, \ldots, \mathrm{t}_{6}\right)=\mathrm{t}_{1}^{2}-\alpha \max \left\{\mathrm{t}_{2}^{2}, \mathrm{t}_{3}^{2}, \mathrm{t}_{4}^{2}\right\}-\beta \max \left\{\frac{\mathrm{t}_{3} \mathrm{t}_{5}}{2}, \frac{\mathrm{t}_{4} \mathrm{t}_{6}}{2}\right\}-\gamma \mathrm{t}_{5} \mathrm{t}_{6} . \\
& \text { Where } \alpha, \beta, \gamma, \geq 0 \text { and } \alpha+\beta+\gamma<1 .
\end{aligned}
$$

Popa et al[16], noticed that Husain and Sehgal [3] type contraction conditions (e.g. [2,9,10,18]) can be deduced from similar implicit relations in addition to all earlier ones if we slightly modified(3.1) as follows:

(3.1)' $\mathrm{F}$ is decreasing in variables $\mathrm{t}_{2}, \ldots, \mathrm{t}_{6}$.

Hereafter, let $\mathrm{F}: \mathrm{R}_{+}^{6} \rightarrow$ Rbe a continuous function which satisfy the conditions (3.1)',(3.2), (3.3), (3.4) and (3.5) and let $\psi$ be the family of such functions F. Some examples of [16].

Example 3.16: Define $F\left(t_{1}, t_{2}, \ldots, t_{6}\right): R_{+}^{6} \rightarrow R$ as

$$
\mathrm{F}\left(\mathrm{t}_{1}, \mathrm{t}_{2}, \ldots, \mathrm{t}_{6}\right)=\mathrm{t}_{1}-\varphi\left(\max \left\{\mathrm{t}_{2}, \mathrm{t}_{3}, \mathrm{t}_{4}, \frac{1}{2}\left(\mathrm{t}_{5}+\mathrm{t}_{6}\right)\right\}\right)
$$

Where $\varphi: R^{+} \rightarrow R^{+}$is an increasing upper semi continuous function with $\varphi(0)=0$ and $\varphi(t)<t$ for each $t>0$. (3.1)': Obvious.

(3.2)((3.3)): Let $\mathrm{u}>0 . \mathrm{F}(\mathrm{u}, \mathrm{v}, \mathrm{v}, \mathrm{u}, \mathrm{u}+\mathrm{v}, 0)=\mathrm{u}-\varphi(\max \{\mathrm{v}, \mathrm{v}, \mathrm{u},(\mathrm{u}+\mathrm{v}) / 2\}) \leq 0$.

If $\mathrm{u} \geq \mathrm{v}$, then $\mathrm{u} \leq \varphi(\mathrm{u})<\mathrm{u}$ which is a contradiction. Hence $\mathrm{u}<\mathrm{v}$ and $\mathrm{u} \leq \mathrm{hv}$

Where $\mathrm{h} \in(0,1)$.

(3.4): Similar argument as in (3.3).

(3.5): $\mathrm{F}(\mathrm{u}, \mathrm{u}, 0,0, \mathrm{u}, \mathrm{u})=\mathrm{u}-\varphi(\max \{\mathrm{u}, 0,0,(\mathrm{u}+\mathrm{u}) / 2\})=\mathrm{u}-\varphi(\mathrm{u})>0$ for all $\mathrm{u}>0$.

Example 3.17: Define $F\left(t_{1}, t_{2}, \ldots, t_{6}\right): R_{+}^{6} \rightarrow R$ as

$$
\mathrm{F}\left(\mathrm{t}_{1}, \mathrm{t}_{2}, \ldots, \mathrm{t}_{6}\right)=\mathrm{t}_{1}-\varphi\left(\mathrm{t}_{2}, \mathrm{t}_{3}, \ldots, \mathrm{t}_{6}\right)
$$

Where $\varphi: \mathrm{R}_{+}^{5} \rightarrow \mathrm{R}^{+}$is an upper semi continuous and non decreasing function in each coordinate variable such that $\varphi(\mathrm{t}, \mathrm{t}, \alpha \mathrm{t}, \beta \mathrm{t}, \gamma \mathrm{t})<\mathrm{t}$ for each $\mathrm{t}>0$ and $\alpha, \beta, \gamma \geq 0$ with

$$
\alpha+\beta+\gamma \leq 3 \text {. }
$$

Example 3.18: Define $F\left(t_{1}, t_{2}, \ldots, t_{6}\right): R_{+}^{6} \rightarrow R$ as

$$
\mathrm{F}\left(\mathrm{t}_{1}, \mathrm{t}_{2}, \ldots, \mathrm{t}_{6}\right)=\mathrm{t}_{1}^{2}-\varphi\left(\mathrm{t}_{2}^{2}, \mathrm{t}_{3} \mathrm{t}_{4}, \mathrm{t}_{5} \mathrm{t}_{6}, \mathrm{t}_{3} \mathrm{t}_{6}, \mathrm{t}_{4} \mathrm{t}_{5}\right)
$$

Where $\varphi: \mathrm{R}_{+}^{5} \rightarrow \mathrm{R}^{+}$is an upper semi continuous and non decreasing function in each coordinate variable such that $\varphi(t, t, \alpha t, \beta t, \gamma t)<t$ for each $\mathrm{t}>0$ and $\alpha, \beta, \gamma \geq 0$ with $\alpha+\beta+\gamma \leq 3$.

Here it may be noticed that all earlier mentioned examples continue to enjoy the format of modified implicit relation as adopted herein.

Now we state our first main result:

\section{Main Result}

4.1 Theorem : L, M, A, B, S and T be self mappings of a metric space (X, d) satisfying the conditions

(4.1.1) $\quad F(d(L x, M y), d(A B x, S T y), d(A B x, L x), d(S T y, M y), d(A B x, M y), d(S T y, L x)) \leq 0$

(4.1.2) $\quad \mathrm{L}(\mathrm{X}) \subseteq \mathrm{ST}(\mathrm{X})$ and $\mathrm{M}(\mathrm{X}) \subseteq \mathrm{AB}(\mathrm{X})$.

If one of $\mathrm{L}(\mathrm{X}), \mathrm{M}(\mathrm{X}), \mathrm{AB}(\mathrm{X})$ or $\mathrm{ST}(\mathrm{X})$ is a complete subspace of $\mathrm{X}$, then

(4.1.3) the pair (L,AB) has a point of coincidence,

(4.1.4) the pair (M, ST) has a point of coincidence.

Moreover, L,M, AB and ST have a unique common fixed point provided both the pairs (L, AB) or (M, ST) is occasionally weakly compatible mappings.

Further if

(A, B), (S,T), (M,T), (L,T), (M,B) are commuting mappings then A,B,S,T,L and $\mathrm{M}$ have a unique common fixed point.

Proof. Since $\mathrm{L}(\mathrm{X}) \subseteq \mathrm{ST}(\mathrm{X})$, for arbitrary point $\mathrm{x}_{0} \in X$ there exists a point $\mathrm{x}_{1} \in X$ such that $\mathrm{Lx}_{0}=\mathrm{STx}_{1}$. Since $\mathrm{M}(\mathrm{X}) \subseteq \mathrm{AB}(\mathrm{X})$, for the point $\mathrm{x}_{1}$, we can choose a point $\mathrm{x}_{2} \in \mathrm{X}$ such that $\mathrm{Mx}_{1}=\mathrm{ABx}_{2}$ and so on. Inductively, we can define a sequence $\left\langle\mathrm{y}_{\mathrm{n}}\right\rangle$ in $\mathrm{X}$ such that (4.1.5) $\quad \mathrm{y}_{2 \mathrm{n}}=\mathrm{Lx}_{2 \mathrm{n}}=\mathrm{STx}_{2 \mathrm{n}+1}$ and $\mathrm{y}_{2 \mathrm{n}+1}=\mathrm{Mx}_{2 \mathrm{n}+1}=\mathrm{ABx}_{2 \mathrm{n}+2} ; \mathrm{n}=0,1,2, \ldots$ From (4.1.1) we have

$$
\mathrm{F}\left(\mathrm{d}\left(\mathrm{Lx}_{2 \mathrm{n}+2}, \mathrm{Mx}_{2 \mathrm{n}+1}\right), \mathrm{d}\left(\mathrm{ABx}_{2 \mathrm{n}+2}, \mathrm{STx}_{2 \mathrm{n}+1}\right), \mathrm{d}\left(\mathrm{ABx}_{2 \mathrm{n}+2}, \mathrm{Lx}_{2 \mathrm{n}+2}\right), \mathrm{d}\left(\mathrm{STx}_{2 \mathrm{n}+1}, \mathrm{Mx}_{2 \mathrm{n}+1}\right),\right.
$$
$\left.\mathrm{d}\left(\mathrm{ABx}_{2 \mathrm{n}+2}, \mathrm{Mx}_{2 \mathrm{n}+1}\right), \mathrm{d}\left(\mathrm{STx}_{2 \mathrm{n}+1}, \mathrm{Lx}_{2 \mathrm{n}+2}\right)\right) \leq 0$

or $F\left(d\left(y_{2 n+2}, y_{2 n+1}\right), d\left(y_{2 n+1}, y_{2 n}\right), d\left(y_{2 n+1}, y_{2 n+2}\right), d\left(y_{2 n}, y_{2 n+1}\right), d\left(y_{2 n+1}, y_{2 n+1}\right), d\left(y_{2 n}, y_{2 n}\right)\right) \leq 0$ 
or $\mathrm{F}\left(\mathrm{d}\left(\mathrm{y}_{2 \mathrm{n}+2}, \mathrm{y}_{2 \mathrm{n}+1}\right), 0, \mathrm{~d}\left(\mathrm{y}_{2 \mathrm{n}+2}, \mathrm{y}_{2 \mathrm{n}+1}\right), 0,0,0\right) \leq 0$

or $\mathrm{F}\left(\mathrm{d}\left(\mathrm{y}_{2 \mathrm{n}+2}, \mathrm{y}_{2 \mathrm{n}+1}\right), 0, \mathrm{~d}\left(\mathrm{y}_{2 \mathrm{n}+2}, \mathrm{y}_{2 \mathrm{n}+1}\right), 0,0, \mathrm{~d}\left(\mathrm{y}_{2 \mathrm{n}+2}, \mathrm{y}_{2 \mathrm{n}+1}\right)\right) \leq 0$

Yielding thereby $\mathrm{d}\left(\mathrm{y}_{2 \mathrm{n}+2}, \mathrm{y}_{2 \mathrm{n}+1}\right)=0$ (due to (3.4)). Similarly, using (3.3) we can show that

$\mathrm{d}\left(\mathrm{y}_{2 \mathrm{n}+1}, \mathrm{y}_{2 \mathrm{n}}\right)=0$. Thus it follows that $\mathrm{d}\left(\mathrm{y}_{\mathrm{n}}, \mathrm{y}_{\mathrm{n}+1}\right)=0$ for every $\mathrm{n} \in \mathrm{N}$.

Let us write $d_{n}=d\left(y_{n}, y_{n+1}\right), n=0,1,2, \ldots$ First we shall prove that $\left\langle d_{n}\right\rangle$ is a non-decreasing sequence in $R^{+}$.

From (4.1.1), we have

$\mathrm{F}\left(\mathrm{d}\left(\mathrm{Lx}_{2 \mathrm{n}}, \mathrm{Mx}_{2 \mathrm{n}+1}\right), \mathrm{d}\left(\mathrm{ABx}_{2 \mathrm{n}}, \mathrm{STx}_{2 \mathrm{n}+1}\right), \mathrm{d}\left(\mathrm{ABx}_{2 \mathrm{n}}, \mathrm{Lx}_{2 \mathrm{n}}\right), \mathrm{d}\left(\mathrm{STx}_{2 \mathrm{n}+1}, \mathrm{Mx}_{2 \mathrm{n}+1}\right)\right.$,

$\left.\mathrm{d}\left(\mathrm{ABx}_{2 \mathrm{n}, \mathrm{Mx}} \mathrm{Mx}_{2 \mathrm{n}+1}\right), \mathrm{d}\left(\mathrm{STx}_{2 \mathrm{n}+1}, \mathrm{Lx}_{2 \mathrm{n}}\right)\right) \leq 0$,

or $F\left(d\left(y_{2 n}, y_{2 n+1}\right), d\left(y_{2 n-1}, y_{2 n}\right), d\left(y_{2 n-1}, y_{2 n}\right), d\left(y_{2 n}, y_{2 n+1}\right), d\left(y_{2 n-1}, y_{2 n+1}\right), d\left(y_{2 n}, y_{2 n}\right)\right) \leq 0$,

or $F\left(d\left(y_{2 n}, y_{2 n+1}\right), d\left(y_{2 n-1}, y_{2 n}\right), d\left(y_{2 n-1}, y_{2 n}\right), d\left(y_{2 n}, y_{2 n+1}\right)\right.$, $\left.\mathrm{d}\left(\mathrm{y}_{2 \mathrm{n}-1}, \mathrm{y}_{2 \mathrm{n}+1}\right)+\mathrm{d}\left(\mathrm{y}_{2 \mathrm{n}-1}, \mathrm{y}_{2 \mathrm{n}}\right)+\mathrm{d}\left(\mathrm{y}_{2 \mathrm{n}+1}, \mathrm{y}_{2 \mathrm{n}}\right), 0\right) \leq 0$

or $F\left(d\left(y_{2 n}, y_{2 n+1}\right), d\left(y_{2 n-1}, y_{2 n}\right), d\left(y_{2 n-1}, y_{2 n}\right), d\left(y_{2 n}, y_{2 n+1}\right), d\left(y_{2 n-1}, y_{2 n}\right)+d\left(y_{2 n}, y_{2 n+1}\right), 0\right) \leq 0$

Implying thereby $d_{2 n} \leq h_{2 n-1}<d_{2 n-1}$ (due to (3.3)). Similarly using (3.4), we have $d_{2 n+1} \leq h d_{2 n}$. Thus $d_{n+1}<d_{n}$ for $n$ $=0,1,2, \ldots$ Now proceeding on the lines of the proof of Lemma $3.2[14, \mathrm{p} .355]$, we can show that

$\mathrm{d}\left(\mathrm{y}_{\mathrm{i}}, \mathrm{y}_{\mathrm{j}}\right)=0$ for $\mathrm{i}, \mathrm{j} \in \mathrm{N}$.

Now we show $<\mathrm{y}_{\mathrm{n}}>$ is a sequence in a metric space (X, d) described by (4.1.5), then

$\lim _{n \rightarrow \infty} d\left(y_{n}, y_{n+1}\right)=0$.

We have $d_{2 n+1} \leq h d_{2 n}$ and $d_{2 n} \leq h d_{2 n-1}$. Therefore, we obtain $d_{n} \leq h^{n} d_{0}$. Hence $\lim _{n \rightarrow \infty} d\left(y_{n,}, y_{n+1}\right)=\lim _{n \rightarrow \infty} d_{n}=0$.

The sequence $\left\langle\mathrm{y}_{\mathrm{n}}\right\rangle$ described by (4.1.5) is a Cauchy sequence.

Since $\lim d\left(y_{n}, y_{n+1}\right)=0$, it is sufficient to show that a subsequence $\left\langle y_{2 n}\right\rangle$ of $\left\langle y_{n}\right\rangle$ is a Cauchy sequence in $X$.

Suppose that $\left\langle\mathrm{y}_{2 \mathrm{n}}>\right.$ is not a Cauchy sequence in $\mathrm{X}$. Then for every $\in>0$ there exists strictly increasing sequences $\left\langle\mathrm{m}_{\mathrm{k}}>,\left\langle\mathrm{n}_{\mathrm{k}}>\right.\right.$ of positive integers such that $\mathrm{k} \leq \mathrm{n}_{\mathrm{k}}<\mathrm{m}_{\mathrm{k}}$ with $\mathrm{d}\left(y_{2 n_{k}-1}, y_{2 m_{k}}\right) \geq \in$ and $\mathrm{d}\left(y_{2 n_{k}}, y_{2 m_{k}-2}\right)<\in$. Now proceeding on the lines of the proof of Lemma 1.3[1] (or Lemma 3.3[14]), we obtain

$\lim _{\mathbf{n} \rightarrow \infty} \mathrm{d}\left(y_{2 n_{k}}, y_{2 m_{k}}\right)=\epsilon, \lim _{\mathbf{n} \rightarrow \infty} \mathrm{d}\left(y_{2 n_{k}}, y_{2 m_{k}-1}\right)=\epsilon, \lim _{\mathbf{n} \rightarrow \infty} \mathrm{d}\left(y_{2 n_{k}+1}, y_{2 m_{k}}\right)=\epsilon$ and

$\lim _{\mathbf{n} \rightarrow \infty} \mathrm{d}\left(y_{2 n_{k}+1}, y_{2 m_{k}-1}\right)=\in$. Now using (4.1.1), we have

$\mathrm{F}\left(\mathrm{d}\left(\mathrm{L} x_{2 m_{k}}, \mathrm{M} x_{2 n_{k}+1}\right), \mathrm{d}\left(\mathrm{AB} x_{2 m_{k}}, \mathrm{ST} x_{2 n_{k}+1}\right), \mathrm{d}\left(\mathrm{AB} x_{2 m_{k}}, \mathrm{~L} x_{2 m_{k}}\right)\right.$, $\mathrm{d}\left(\mathrm{M} x_{2 n_{k}+1}, \mathrm{ST} x_{2 n_{k}+1}\right), \mathrm{d}\left(\mathrm{AB} x_{2 m_{k}}, \mathrm{M} x_{2 n_{k}+1}\right), \mathrm{d}\left(\mathrm{ST} x_{2 n_{k}+1}, \mathrm{~L} x_{2 m_{k}}\right) \leq 0$

$\operatorname{orF}\left(\mathrm{d}\left(y_{2 m_{k}}, y_{2 n_{k}+1}\right), \mathrm{d}\left(y_{2 m_{k}-1}, y_{2 n_{k}}\right), \mathrm{d}\left(y_{2 m_{k}-1}, y_{2 m_{k}}\right)\right.$, $\left.\mathrm{d}\left(y_{2 n_{k}}, y_{2 n_{k}+1}\right), \mathrm{d}\left(y_{2 m_{k}-1}, y_{2 n_{k}+1}\right), \mathrm{d}\left(y_{2 n_{k}}, y_{2 m_{k}}\right)\right) \leq 0$.

Letting $\mathrm{n} \rightarrow \infty$, we have $\mathrm{F}(\in, \in, 0,0, \in, \in) \leq 0$, which is a contradiction to (3.5).

Therefore $\left\langle\mathrm{y}_{2 \mathrm{n}}\right\rangle$ is a Cauchy sequence.

Suppose that $A B(X)$ is a complete subspace of $X$ then the subsequence $\left\{y_{2 n+1}\right\}$ which is contain in $A B(X)$. We must have a limit $\mathrm{z}$ in $\mathrm{AB}(\mathrm{X})$.

As $\left\langle y_{n}\right\rangle$ is a Cauchy sequence containing a convergent subsequence $\left\langle y_{2 n+1}\right\rangle$, therefore $\left\langle y_{n}\right\rangle$ also

converges implying thereby the convergence of the subsequence $\left\langle\mathrm{y}_{2 \mathrm{n}}\right\rangle$, i.e. $\operatorname{limLx} \mathrm{x}_{2 \mathrm{n}}=\operatorname{limMx_{2n+1}}=\lim \mathrm{STx}_{2 \mathrm{n}+1}=$ $\lim \mathrm{ABx}_{2 \mathrm{n}+2}=\mathrm{z}$. Let $\mathrm{u} \in(\mathrm{AB})^{-1}(\mathrm{z})$, then

$\mathrm{ABu}=\mathrm{z}$. If $\mathrm{Lu} \neq \mathrm{z}$, then using (4.1.1), we have

$\mathrm{F}\left(\mathrm{d}\left(\mathrm{Lu}, \mathrm{Mx}_{2 \mathrm{n}-1}\right), \mathrm{d}\left(\mathrm{ABu}, \mathrm{STx}_{2 \mathrm{n}-1}\right), \mathrm{d}(\mathrm{ABu}, \mathrm{Lu}), \mathrm{d}\left(\mathrm{STx}_{2 \mathrm{n}-1}, \mathrm{Mx}_{2 \mathrm{n}-1}\right)\right.$,

$\left.\mathrm{d}\left(\mathrm{ABu}, \mathrm{Mx}_{2 \mathrm{n}-1}\right), \mathrm{d}\left(\mathrm{STx}_{2 \mathrm{n}-1}, \mathrm{Lu}\right)\right) \leq 0$

which on letting $n \rightarrow \infty$, reduces to

$\mathrm{F}(\mathrm{d}(\mathrm{Lu}, \mathrm{z}), \mathrm{d}(\mathrm{z}, \mathrm{z}), \mathrm{d}(\mathrm{z}, \mathrm{Lu}) ; \mathrm{d}(\mathrm{z}, \mathrm{z}), \mathrm{d}(\mathrm{z}, \mathrm{z}), \mathrm{d}(\mathrm{z}, \mathrm{Lu})) \leq 0$

or

$\mathrm{F}(\mathrm{d}(\mathrm{Lu}, \mathrm{z}), 0, \mathrm{~d}(\mathrm{z}, \mathrm{Lu}), 0,0, \mathrm{~d}(\mathrm{z}, \mathrm{Lu})) \leq 0$

implying thereby $\mathrm{d}(\mathrm{z}, \mathrm{Lu})=0$ (due to (3.4)).

Hence $\mathrm{z}=\mathrm{Lu}=\mathrm{ABu}$.

Since $\mathrm{L}(\mathrm{X}) \subseteq \mathrm{ST}(\mathrm{X})$, there exists $\mathrm{v} \in(\mathrm{ST})^{-1}(\mathrm{z})$ such that $\mathrm{STv}=\mathrm{z}$. By (4.1.1), we have $\mathrm{F}(\mathrm{d}(\mathrm{Lu}, \mathrm{Mv}), \mathrm{d}(\mathrm{ABu}, \mathrm{STv}), \mathrm{d}(\mathrm{ABu}, \mathrm{Lu}), \mathrm{d}(\mathrm{STv}, \mathrm{Mv}), \mathrm{d}(\mathrm{ABu}, \mathrm{Mv}), \mathrm{d}(\mathrm{STv}, \mathrm{Lu})) \leq 0$

or $\quad \mathrm{F}(\mathrm{d}(\mathrm{z}, \mathrm{Mv}), 0,0, \mathrm{~d}(\mathrm{z}, \mathrm{Mv}), \mathrm{d}(\mathrm{z}, \mathrm{Mv}), 0) \leq 0$

yielding thereby $\mathrm{d}(\mathrm{z}, \mathrm{Mv})=0$ (due to (3.3)). Therefore

$\mathrm{z}=\mathrm{Mv}$. Hence $\mathrm{Lu}=\mathrm{ABu}=\mathrm{Mv}=\mathrm{STv}=\mathrm{z}$ which establishes (4.1.3) and (4.1.4).

If one assumes that $\mathrm{ST}(\mathrm{X})$ is a complete subspace of $\mathrm{X}$, then analogous arguments establish (4.1.3) and (4.1.4). The remaining two cases also pertain essentially to the previous cases. Indeed, if $L(X)$ is complete, then $z \in$ $\mathrm{L}(\mathrm{X}) \subseteq \mathrm{ST}(\mathrm{X})$. Similarly if $\mathrm{M}(\mathrm{X})$ is complete, then $\mathrm{z} \in \mathrm{M}(\mathrm{X}) \subseteq \mathrm{S}(\mathrm{X})$. Thus in all cases, (4.1.3) and (4.1.4) are completely established. 
Suppose $\mathrm{L}$ and $\mathrm{AB}$ are occasionally weakly compatible.

By occasionally weakly compatible of $\mathrm{L}, \mathrm{AB}$ gives $\mathrm{Lu}=\mathrm{ABu}$ and $\mathrm{L}(\mathrm{AB}) \mathrm{u}=(\mathrm{AB}) \mathrm{Lu}$.

Occasionally weakly compatible of $\mathrm{M}$, ST gives $\mathrm{Mv}=\mathrm{STv}$ and $\mathrm{M}(\mathrm{ST}) \mathrm{v}=(\mathrm{ST}) \mathrm{Mv}$.

$\mathrm{L}(\mathrm{AB}) \mathrm{u}=(\mathrm{AB}) \mathrm{Lu}$ and $\mathrm{LLu}=\mathrm{L}(\mathrm{AB}) \mathrm{u}=(\mathrm{AB}) \mathrm{Lu}=(\mathrm{AB})(\mathrm{AB}) \mathrm{u}$.

We have MMv $=\mathrm{M}(\mathrm{ST}) \mathrm{v}=(\mathrm{ST}) \mathrm{Mv}=(\mathrm{ST})(\mathrm{ST}) \mathrm{v}$.

Form (4.1.1) $\mathrm{F}(\mathrm{d}(\mathrm{LLu}, \mathrm{Mv}), \mathrm{d}((\mathrm{AB}) \mathrm{Lu}, \mathrm{STv}), \mathrm{d}((\mathrm{AB}) \mathrm{Lu}, \mathrm{LLu})$, $\mathrm{d}(\mathrm{STv}, \mathrm{Mv}), \mathrm{d}((\mathrm{AB}) \mathrm{Lu}, \mathrm{Mv}), \mathrm{d}(\mathrm{STv} ; \mathrm{LLu})) \leq 0$

or $\mathrm{F}(\mathrm{d}(\mathrm{LLu}, \mathrm{Mv}), \mathrm{d}(\mathrm{LLu}, \mathrm{Mv}), 0,0, \mathrm{~d}(\mathrm{LLu}, \mathrm{Mv}), \mathrm{d}(\mathrm{LLu}, \mathrm{Mv})) \leq 0$.

Contradition to (3.5) if $\mathrm{d}(\mathrm{LLu}, \mathrm{Mv})>0$.

Hence $\mathrm{LLu}=\mathrm{Mv}=\mathrm{Lu}$. So $\mathrm{Lu}=\mathrm{LLu}=(\mathrm{AB}) \mathrm{Lu}$.

Therefore $\mathrm{Lu}$ is a common fixed point of $\mathrm{L}$ and $\mathrm{AB}$.

Similarlly we can prove that $\mathrm{Mv}(=\mathrm{Lu})$ is a common fixed point of $\mathrm{M}$ and ST.

To Prove Uniqueness of $\mathrm{Lu}$

Suppose that $\mathrm{Lu}$ andLw, $\mathrm{Lu}=\mathrm{Lw}$ are common fixed point of $\mathrm{L}, \mathrm{M}, \mathrm{ST}$ and $\mathrm{AB}$ by (4.1.1)

$\mathrm{F}(\mathrm{d}(\mathrm{LLu}, \mathrm{MLw}), \mathrm{d}((\mathrm{AB}) \mathrm{Lu},(\mathrm{ST}) \mathrm{Lw}), \mathrm{d}(\mathrm{LLu}, \mathrm{ABLu})$, $\mathrm{d}(\mathrm{MLw}, \mathrm{STLw}), \mathrm{d}(\mathrm{LLu},(\mathrm{ST}) \mathrm{Lw}), \mathrm{d}(\mathrm{MLw}, \mathrm{ABLu})) \leq 0$

or $\mathrm{F}(\mathrm{d}(\mathrm{Lu}, \mathrm{Lw}, \mathrm{a}), \mathrm{d}(\mathrm{Lu}, \mathrm{Lw}, \mathrm{a})), 0,0, \mathrm{~d}(\mathrm{Lu}, \mathrm{Lw}, \mathrm{a}), \mathrm{d}(\mathrm{Lu}, \mathrm{Lw}, \mathrm{a})) \leq 0$.

Which shows $\mathrm{Lu}=\mathrm{Lw}$. But $\mathrm{Lu}=\mathrm{z}$, so $\mathrm{z}$ is the common fixed point of $\mathrm{L}, \mathrm{M}, \mathrm{ST}$ and $\mathrm{AB}$.

Finally we need to show that $\mathrm{z}$ is a common fixed point of $\mathrm{A}, \mathrm{B}, \mathrm{L}, \mathrm{M}, \mathrm{S}$ and $\mathrm{T}$.

Since $(A, B),(A, L),(B, L)$ are commutative

$\mathrm{Az}=\mathrm{A}(\mathrm{ABz})=\mathrm{A}(\mathrm{BAz})=(\mathrm{AB})(\mathrm{Az}) ; \mathrm{Az}=\mathrm{ALz}=\mathrm{LAz}$

$\mathrm{Bz}=\mathrm{B}(\mathrm{ABz})=(\mathrm{BA})(\mathrm{Bz})=(\mathrm{AB})(\mathrm{Bz}) ; \mathrm{Bz}=\mathrm{BLz}=\mathrm{LBz}$

Which shows that $\mathrm{Az}, \mathrm{Bz}$ are common fixed points of $(\mathrm{AB}, \mathrm{L})$ yielding there by $\mathrm{Az}=\mathrm{z}=\mathrm{Bz}=\mathrm{Lz}=\mathrm{ABz}$ in the view of uniqueness of common fixed point of the pairs $(\mathrm{AB}, \mathrm{L})$.

Similarly using the, commutativity of $(\mathrm{S}, \mathrm{T}),(\mathrm{S}, \mathrm{M})$ and $(\mathrm{T}, \mathrm{M})$ it can be shown that

$\mathrm{Sz}=\mathrm{z}=\mathrm{Tz}=\mathrm{Mz}=\mathrm{STz}$. Now, we need to show that $\mathrm{Az}=\mathrm{Sz}(\mathrm{Bz}=\mathrm{Tz})$ also remains a common fixed point of both the pairs $(\mathrm{AB}, \mathrm{L})$ and $(\mathrm{ST}, \mathrm{M})$ from 4.1.1 we can easily prove $\mathrm{Az}=\mathrm{Sz}$ and $\mathrm{Bz}=\mathrm{Tz}$. Which shows $\mathrm{z}$ is a common fixed point of A, B, L, M, S and T.

4.1.6 Corollary : The conclusions of Theorem 4.1 remain true if (for all $\mathrm{x}, \mathrm{y} \in \mathrm{X}$ ) implicit relation (4.1.1) is replaced by any one of the following.

(4.1.7) $\quad d(L x, M y) \leq k \max \{d(A B x, S T y), d(A B x, L x), d(S T y, M y)$, $\left.\frac{1}{2}(\mathrm{~d}(\mathrm{ABx}, \mathrm{My})+\mathrm{d}(\mathrm{STy}, \mathrm{Lx}))\right\}$, where $\mathrm{k} \in(0,1)$.

(4.1.8) $\quad \mathrm{d}^{2}(\mathrm{Lx}, \mathrm{My}) \leq \mathrm{d}(\mathrm{Lx}, \mathrm{My})[\alpha \mathrm{d}(\mathrm{ABx}, \mathrm{STy})+\beta \mathrm{d}(\mathrm{ABx}, \mathrm{Lx})+\gamma \mathrm{d}(\mathrm{STy}, \mathrm{My})]+\eta \mathrm{d}(\mathrm{ABx}, \mathrm{My}) \cdot \mathrm{d}(\mathrm{STy} ; \mathrm{Lx})$ Where $\alpha>0 ; \beta, \gamma, \eta \geq 0, \alpha+\beta+\gamma<1$ and $\alpha+\eta<1$.

$\mathrm{d}^{3}(\mathrm{Lx}, \mathrm{My}) \leq \alpha \mathrm{d}^{2}(\mathrm{Lx}, \mathrm{My}) \mathrm{d}(\mathrm{ABx}, \mathrm{STy})+\beta \mathrm{d}(\mathrm{Lx}, \mathrm{My}) \mathrm{d}(\mathrm{ABx}, \mathrm{Lx}) \mathrm{d}(\mathrm{STy}, \mathrm{My})$

$+\gamma \mathrm{d}^{2}(\mathrm{ABx}, \mathrm{My}) \mathrm{d}(\mathrm{STy}, \mathrm{Lx})+\eta \mathrm{d}(\mathrm{ABx}, \mathrm{My}) \mathrm{d}^{2}(\mathrm{STy}, \mathrm{Lx})$

Where $\alpha>0 ; \beta, \gamma, \eta \geq 0, \alpha+\beta<1$ and $\alpha+\eta+\gamma<1$.

$\mathrm{d}^{3}(\mathrm{Lx}, \mathrm{My}) \leq \alpha \frac{d^{2}(A B x, L x) d^{2}(S T y, M y)+d^{2}(A B x, L y) d^{2}(S T y, L x)}{1+d(A B x, S T y)+d(A B x, L x)+d(S T y, M y)}$

Where $\alpha \in(0,1)$.

$\mathrm{d}^{2}(\mathrm{Lx}, \mathrm{My}) \leq \alpha \mathrm{d}^{2}(\mathrm{ABx}, \mathrm{STy})+\beta \frac{d(A B x, M y) d(S T y, L x)}{1+d^{2}(A B x, L x)+d^{2}(S T y, M y)}$

Where $\alpha>0, \beta \geq 0$ and $\alpha+\beta<1$.

$$
\begin{array}{r}
\mathrm{d}(\mathrm{Lx}, \mathrm{My}) \leq \mathrm{a}_{1} \frac{d^{2}(A B x, L x)+d^{2}(S T y, M y)}{d(A B x, L x)+d(S T y, M y)}+\mathrm{a}_{2} \mathrm{~d}(\mathrm{ABx}, \mathrm{STy}) \\
+\mathrm{a}_{3}(\mathrm{~d}(\mathrm{ABx}, \mathrm{My})+\mathrm{d}(\mathrm{STy}, \mathrm{Lx}))
\end{array}
$$

Wherea $a_{i} \geq 0$ with at least one $a_{i}$ non zero and $a_{1}+a_{2}+2 a_{3}<1$.

$\mathrm{d}(\mathrm{Lx}, \mathrm{My}) \leq \alpha \mathrm{d}(\mathrm{ABx}, \mathrm{STy})$

$$
+\frac{\beta d(A B x, L x) d(S T y, M y)+\gamma d(A B x, M y) d(S T y, L x)}{d(A B x, L x)+d(S T y, M y)}
$$

Where $\alpha, \beta, \gamma \geq 0$ such that $1<2 \alpha+\beta<2$.

$\mathrm{d}(\mathrm{Lx}, \mathrm{My}) \leq \mathrm{a}_{1} \mathrm{~d}(\mathrm{ABx}, \mathrm{STy})+\mathrm{a}_{2} \mathrm{~d}(\mathrm{ABx}, \mathrm{Lx})+\mathrm{a}_{3} \mathrm{~d}(\mathrm{STy}, \mathrm{My})+\mathrm{a}_{4} \mathrm{~d}(\mathrm{ABx}, \mathrm{My})$

$$
+\mathrm{a}_{5} \mathrm{~d}(\mathrm{STy}, \mathrm{Lx}), \quad \text { where } \sum_{i=1}^{5} a_{i}<1
$$

$\mathrm{d}(\mathrm{Lx}, \mathrm{My}) \leq \alpha[\beta \max \{\mathrm{d}(\mathrm{ABx}, \mathrm{STy}), \mathrm{d}(\mathrm{ABx}, \mathrm{Lx}), \mathrm{d}(\mathrm{STy}, \mathrm{My})$,

$$
\left.\frac{1}{2}(\mathrm{~d}(\mathrm{ABx}, \mathrm{My})+\mathrm{d}(\mathrm{STy}, \mathrm{Lx}))\right\}+(1-\beta)
$$


$\left.\left.\left[\max d^{2}(\mathrm{ABx}, \mathrm{STy}), \mathrm{d}(\mathrm{ABx}, \mathrm{Lx}) \mathrm{d}(\mathrm{STy}, \mathrm{My}), \mathrm{d}(\mathrm{ABx}, \mathrm{My}) \mathrm{d}(\mathrm{STy}, \mathrm{Lx}), \frac{d(A B x, L x) d(S T y, L x)}{2}, \frac{d(S T y, M y) d(A B x, M y)}{2}\right\}\right]^{\frac{1}{2}}\right]$ Where $\alpha \in(0,1)$ and $0 \leq \beta \leq 1$.

$$
\mathrm{d}^{2}(\mathrm{Lx}, \mathrm{My}) \leq \alpha \max \left\{\mathrm{d}^{2}(\mathrm{ABx}, \mathrm{STy}), \mathrm{d}^{2}(\mathrm{ABx}, \mathrm{Lx}), \mathrm{d}^{2}(\mathrm{STy}, \mathrm{My})\right\}
$$

$$
\begin{aligned}
& +\beta \max \left\{\frac{d(A B x, L x) d(A B x, M y)}{2}, \frac{d(S T y, M y) d(S T y, L x)}{2}\right\} \\
& +\gamma \mathrm{d}(\mathrm{ABx}, \mathrm{My}) \mathrm{d}(\mathrm{ST} \mathrm{T}, \mathrm{Lx})
\end{aligned}
$$

Where $\alpha, \beta, \gamma \geq 0$ and $\alpha+\beta+\gamma<1$.

$\mathrm{d}(\mathrm{Lx}, \mathrm{My}) \leq \varphi(\max \{\mathrm{d}(\mathrm{ABx}, \mathrm{STy}), \mathrm{d}(\mathrm{ABx}, \mathrm{Lx}), \mathrm{d}(\mathrm{STy}, \mathrm{My})$,

$\left.\left.\frac{1}{2}[\mathrm{~d}(\mathrm{ABx}, \mathrm{My})+\mathrm{d}(\mathrm{STy}, \mathrm{Lx})]\right\}\right)$

Where $\varphi: R^{+} \rightarrow R^{+}$is an upper semicontinuous and increasing function with $\varphi(0)=0$ and $\varphi(t)<t$ for each $t>0$. (4.1.18) $\mathrm{d}(\mathrm{Lx}, \mathrm{My}) \leq \varphi(\mathrm{d}(\mathrm{ABx}, \mathrm{STy}), \mathrm{d}(\mathrm{ABx}, \mathrm{Lx}), \mathrm{d}(\mathrm{STy}, \mathrm{By}), \mathrm{d}(\mathrm{ABx}, \mathrm{My}), \mathrm{d}(\mathrm{STy}, \mathrm{Lx}))$

where $\varphi: \mathrm{R}_{+}^{5} \rightarrow \mathrm{R}^{+}$is an upper semicontinuous and nondecreasing function in each coordinate variable such that $\varphi(\mathrm{t}, \mathrm{t}, \alpha \mathrm{t}, \beta \mathrm{t}, \gamma \mathrm{t})<\mathrm{t}$ for each $\mathrm{t}>0$ and $\alpha, \beta, \gamma \geq 0$

with $\alpha+\beta+\gamma \leq 3$

(4.1.19) $\quad d^{2}(L x, M y) \leq \varphi\left(d^{2}(A B x, S T y), d(A B x, L x) d(S T y, M y)\right.$,

$$
\begin{aligned}
& \text { d(ABx,My)d(STy,Lx),d(ABx, Lx)d(STy, Lx), } \\
& \text { d(STy, My)d(ABx,My)) }
\end{aligned}
$$

where $\varphi: \mathrm{R}_{+}^{5} \rightarrow \mathrm{R}^{+}$is an upper semicontinuous and nondecreasing function in each coordinate variable such that $\varphi(\mathrm{t}, \mathrm{t}, \alpha \mathrm{t}, \beta \mathrm{t}, \gamma \mathrm{t})<\mathrm{t}$ for each $\mathrm{t}>0$ and $\alpha, \beta, \gamma \geq 0$

with $\alpha+\beta+\gamma \leq 3$

Proof. The proof follows from Theorem 4.1 and Examples 3.6 to 3.18 the above.

Put $B=T=I_{x}$ in corollary 4.1.6, we get result in four self maps.

If we put $B=T=I_{x}$ where $I_{x}$ identity self map on $X$ in theorem 4.1 then we get the following result.

4.1.20 Corollary: L,M,A and $S$ be self mappings of a metric space $(X, d)$ satisfying the conditions $\mathrm{F}(\mathrm{d}(\mathrm{Lx}, \mathrm{My}), \mathrm{d}(\mathrm{Ax}, \mathrm{Sy}), \mathrm{d}(\mathrm{Ax}, \mathrm{Lx}), \mathrm{d}(\mathrm{Sy}, \mathrm{My}), \mathrm{d}(\mathrm{Ax}, \mathrm{My}), \mathrm{d}(\mathrm{Sy}, \mathrm{Lx})) \leq 0$

for all $\mathrm{x}, \mathrm{y} \in \mathrm{X}$ where $\mathrm{F}$ enjoys the property $(3.5)$ and $\mathrm{L}(\mathrm{X}) \subseteq \mathrm{S}(\mathrm{X})$ and $\mathrm{M}(\mathrm{X}) \subseteq \mathrm{A}(\mathrm{X})$.

If one of $\mathrm{L}(\mathrm{X}), \mathrm{M}(\mathrm{X}), \mathrm{A}(\mathrm{X})$ or $\mathrm{S}(\mathrm{X})$ is a complete subspace of $\mathrm{X}$, then

(4.1.21) the pair $(\mathrm{L}, \mathrm{A})$ has a point of coincidence,

(4.1.22) the pair $(\mathrm{M}, \mathrm{S})$ has a point of coincidence.

Moreover, L,M,A and Shave a unique common fixed point provided both the pairs (L, A) or (M, S) is occasionally weakly compatible mappings.

Taking $\mathrm{L}=\mathrm{M}$ and $\mathrm{T}=\mathrm{B}=\mathrm{I}_{\mathrm{X}}$ in theorem 4.1 we get the following corollary.

4.1.23 Corollary: $L, A$ and $S$ be self mappings of a metric space $(X, d)$ satisfying the conditions $\mathrm{F}(\mathrm{d}(\mathrm{Lx}, \mathrm{Ly}), \mathrm{d}(\mathrm{Ax}, \mathrm{Sy}), \mathrm{d}(\mathrm{Ax}, \mathrm{Lx}), \mathrm{d}(\mathrm{Sy}, \mathrm{Ly}), \mathrm{d}(\mathrm{Ax}, \mathrm{Ly}), \mathrm{d}(\mathrm{Sy}, \mathrm{Lx})) \leq 0$ for all $\mathrm{x}, \mathrm{y} \in \mathrm{X}$ where $\mathrm{F}$ enjoys the property $(3.5)$ and $\mathrm{L}(\mathrm{X}) \subseteq \mathrm{S}(\mathrm{X})$ and $\mathrm{L}(\mathrm{X}) \subseteq \mathrm{A}(\mathrm{X})$.. If one of $\mathrm{L}(\mathrm{X}), \mathrm{A}(\mathrm{X})$ or $\mathrm{S}(\mathrm{X})$ is a complete subspace of $\mathrm{X}$, then (4.1.24) the pair $(\mathrm{L}, \mathrm{A})$ has a point of coincidence,

(4.1.25) the pair $(\mathrm{L}, \mathrm{S})$ has a point of coincidence.

Moreover, L, A and Shave a unique common fixed point provided both the pairs (L, A) or $(\mathrm{L}, \mathrm{S})$ is occasionally weakly compatible mappings.

Taking $\mathrm{A}=\mathrm{S}$ and $\mathrm{T}=\mathrm{B}=\mathrm{I}_{\mathrm{X}}$ in theorem 4.1 we get the following corollary.

4.1.26 Corollary: L,M and $\mathrm{S}$ be self mappings of a metric space $(X, d)$ satisfying the conditions $\mathrm{F}(\mathrm{d}(\mathrm{Lx}, \mathrm{My}), \mathrm{d}(\mathrm{Sx}, \mathrm{Sy}), \mathrm{d}(\mathrm{Ax}, \mathrm{Lx}), \mathrm{d}(\mathrm{Sy}, \mathrm{My}), \mathrm{d}(\mathrm{Sx}, \mathrm{My}), \mathrm{d}(\mathrm{Sy}, \mathrm{Lx})) \leq 0$

for all $\mathrm{x}, \mathrm{y} \in \mathrm{X}$ where $\mathrm{F}$ enjoys the property (3.5) and $\mathrm{L}(X) \subseteq \mathrm{S}(X)$ and $\mathrm{M}(X) \subseteq \mathrm{S}(X)$.

If one of $\mathrm{L}(\mathrm{X}), \mathrm{M}(\mathrm{X})$ or $\mathrm{S}(\mathrm{X})$ is a complete subspace of $\mathrm{X}$, then

(4.1.27) the pair $(\mathrm{L}, \mathrm{S})$ has a point of coincidence,

(4.1.28) the pair $(\mathrm{M}, \mathrm{S})$ has a point of coincidence.

Moreover, L,M and Shave a unique common fixed point provided both the pairs $(\mathrm{L}, \mathrm{S})$ or $(\mathrm{M}, \mathrm{S})$ is occasionally weakly compatible mappings.

Taking $\mathrm{L}=\mathrm{M}, \mathrm{A}=\mathrm{S}$ and $\mathrm{T}=\mathrm{B}=\mathrm{I}_{\mathrm{X}}$ in theorem 4.1 we get the following corollary. 
4.1.29 Corollary: $L$ and $A$ be self mappings of a metric space $(X, d)$ satisfying the conditions $\mathrm{F}(\mathrm{d}(\mathrm{Lx}, \mathrm{Ly}), \mathrm{d}(\mathrm{Ax}, \mathrm{Ay}), \mathrm{d}(\mathrm{Ax}, \mathrm{Lx}), \mathrm{d}(\mathrm{Ay}, \mathrm{Ly}), \mathrm{d}(\mathrm{Ax}, \mathrm{Ly}), \mathrm{d}(\mathrm{Ay}, \mathrm{Lx})) \leq 0$

for all $\mathrm{x}, \mathrm{y} \in \mathrm{X}$ where $\mathrm{F}$ enjoys the property (3.5) and $\mathrm{L}(\mathrm{X}) \subseteq \mathrm{A}(\mathrm{X})$. If one of $\mathrm{L}(\mathrm{X})$ or $\mathrm{A}(\mathrm{X})$ is a complete subspace of $X$, then

(4.1.30) the pair (L,A) has a point of coincidence,

Moreover, $\mathrm{L}$ and Ahave a unique common fixed point provided the pair $(\mathrm{L}, \mathrm{A})$ is occasionally weakly

compatible mappings.

\section{References:}

[1] Y. J. Cho, M. S. Khan and S. L. Singh, Common fixed points of weakly commuting mappings, Univ. u NovomSaduZb. Rad. Prirod.-Mat. Fak. Ser. Mat. 18 (1988), no. 1, 129-142.

[2] J. Danes, Two fixed point theorems in topological and metric spaces, Bull. Austral.Math. Soc. 14 (1976), no. 2, $259-265$.

[3] S. A. Husain and V. M. Sehgal, On common fixed points for a family of mappings, Bull. Austral.Math. Soc. 13 (1975), no. 2, 261 267.

[4] G. Jungck, Compatible mappings and common fixed points, Internat. J. Math and Math Sci., 9(4)(1986), $771-779$.

[5] G.Jungck, P.P. Murthy, Y.J.Cho., Compatible mappings of type (A) and common fixed point theorems, Math. Japonica, 38( 2) (1993), 381-390.

[6] G.Jungck, and B.E. Rhoades, Fixed point for set valued functions without continuity, Indian J.Pure Appl. Math, 29(3)(1998), 227238.

[7] G. Jungck and B. E. Rhoades, Fixed Point Theorems for occasionally weakly compatible Mappings, Fixed Point Theory, Vol 7,(2)(2006), No. 287-296.

[8] R. Kannan, Some results on fixed points, Bull. Cal. Math. Soc., 60(1968), 71-76.

[9] M. S. Khan and M. Imdad, A common fixed point theorem for a class of mappings, Indian J. Pure Appl. Math. 14 (1983), no. 10, $1220-1227$.

[10] S. V. R. Naidu and J. Rajendra Prasad, Common fixed points for four self-maps on a metric space, Indian J. Pure Appl. Math. 16 (1985), no. 10, 1089-1103.

[11] H.K.Pathak, Cho, Y.J., Chang, S.S. and Kang S.M., Compatible mappings of type(P) and fixed point theorems in metric spaces and probabilistic metric spaces, Novi sad. J. Math.,26(2)(1996),87-109.

[12] H.K.Pathak, Y. J. Cho, S.M. Kang and B. Madharia, Compatible mappings of type (C) and common fixed point theorems of gregus type, Demonstr. Math., 31(3) (1998), 499-518.

[13] H.K.Pathak and M.S. Khan, Compatible mappings of type(B) and common fixed point theorems of gregus type, Czech. Math. J., 45(120) (1995), 685-698.

[14] H. K. Pathak, S. M. Kang and J. H. Baek, Weak compatible mappings of type (A) and common fixed points, Kyungpook Math. J. 35 (1995), no. 2, 345-359.

[15] V. Popa, Some fixed point theorems for compatible mappings satisfying an implicit relation, Demonstratio Math. 32 (1999), no. 1, $157-163$.

[16] V. Popa, M. Imdad and Javid Ali, Using implicit relations to prove unified fixed point theorem in metric \& 2-metric spaces, Bull.Malayas.Math.Soc.(2) 33(1)(2010),105-120.

[17] S. Sessa, On a weak commutativity condition of mappings in a fixed point considerations, Publ. Inst. Math. (Beogard), 32(46) (1982), 149-153.

[18] S. P. Singh and B. A. Meade, On common fixed point theorems, Bull. Austral.Math.Soc.16(1977), no. 1, 49-53. 